\title{
Generation of stable and breathing flat-top solitons via Raman assisted four wave mixing in microresonators
}

\author{
Shunyu Yao $\odot,{ }^{1}$ Chengying Bao, ${ }^{2}$ Pan Wang, ${ }^{1}$ and Changxi Yang ${ }^{1, *}$ \\ ${ }^{1}$ State Key Laboratory of Precision Measurement Technology and Instruments, Department of Precision Instruments, Tsinghua University, \\ Beijing 100084, China \\ ${ }^{2}$ T. J. Watson Laboratory of Applied Physics, California Institute of Technology, Pasadena, California 91125, USA
}

(Received 16 August 2019; accepted 27 January 2020; published 19 February 2020)

\begin{abstract}
Flat-top-soliton (or platicon) dynamics in coherently pumped normal dispersion microresonators is important for both fundamental nonlinear physics and microcomb generation in the visible band. Here we numerically investigate the platicon generation that is initiated via Raman assisted four wave mixing instead of mode interaction. To show the possibility of generating coherent combs in the visible band, we design an aluminum nitride (AlN) microresonator with normal dispersion and investigate the comb generation dynamics in simulations. Stable platicon Kerr combs can be generated in this AlN microresonator using a 780-nm pump. Moreover, we also observe a breather platicon dynamics induced by the narrow Raman gain spectrum of crystalline AlN, which shows distinct dynamics from the dark soliton breathers reported in previous work that are dominated by Kerr effect. A phase diagram bearing the influence of the pump detuning and pump power on the breathing dynamics of the breather platicon is also computed. Furthermore, a transition to chaotic breathing is numerically observed.
\end{abstract}

DOI: 10.1103/PhysRevA.101.023833

\section{INTRODUCTION}

Kerr frequency combs generated in continuous wave (cw) laser driven microresonators via cascade four wave mixing (FWM) have attracted widespread interest over the past decade [1-8]. They offer high comb line power, compactness, broad bandwidth, and complementary metal-oxidesemiconductor compatibility. Particularly, highly coherent Kerr frequency combs have been used in applications such as arbitrary waveform generation [9], optical atomic clocks [10], optical communication [11], spectroscopy [12,13], and laser ranging systems $[14,15]$. In general, coherent Kerr combs are related to stable localized structures, such as Turing patterns [16], bright dissipative cavity solitons [17,18], soliton crystals [19], or platicons (dark solitons) [6,20]. Most of the demonstrated microcombs operate in the near-infrared band. However, microcomb generation in the visible is also highly demanded for applications in optical atomic clocks, biomedical imaging, frequency metrology, and astronomical calibration [21,22]. Platicon generation in nonlinear microresonators provides an important route to realize microcomb generation in the visible band, as it can be generated in microresonators with normal group velocity dispersion (GVD) [20]. Moreover, comb generation in the normal dispersion regime may provide higher pump power conversion efficiency $[6,23]$. However, the initialization of platicon generation usually requires assistance from mode interaction [6,20], the properties of which are hard to control in experiments. On the other hand, stimulated Raman scattering (SRS) effect can exist in microresonators and has non-negligible impact on the Kerr comb generation

*cxyang@tsinghua.edu.cn dynamics [18,24-26]. Particularly, the Raman gain for crystalline microresonators such as magnesium fluoride, silicon, diamond, and aluminum nitride (AlN) is strong and narrow. Previous work focused on single mode Raman lasing rather than Kerr combs in those microresonators [27-29], and there is competition between SRS and degenerate FWM [30,31]. When the pump power increases or the detuning between pump frequency and cavity resonance varies, sidebands around the pump line and Raman laser may rise [29]. The generation of these sidebands, which are also called Kerr-Raman combs, can be attributed to the Raman assisted four wave mixing (RFWM) process. It is notable that the RFWM process can occur in normal dispersion, providing another opportunity to generate platicons [32,33]. Although the impact of SRS on mode-interaction induced platicons has been investigated [31], whether platicons can be initiated via a narrow Raman gain spectrum without mode interaction remains an open question.

In addition, due to the tight confinement and power enhancement in high-Q microresonators, a rich panel of dynamical instabilities can be obtained [16,34]. For example, breather dynamics have attracted widespread interest [25,35-37]. Similar to Akhmediev breathers in a conservative system (e.g., optical fibers), bright breather solitons in microresonators induce the Fermi-Pasta-Ulam (FPU) recurrence, which exhibits periodical power exchange between comb lines at the center and the wing [25]. Investigation on breather dynamics is not only of fundamental interests, but also important for applications, as breathers constitute an important operation regime in microcombs [38]. Breathers can exist not only in the anomalous dispersion regime (bright soliton breathers) $[25,36,37]$ but also in the normal dispersion regime (dark soliton breathers) [35]. The effect of broadband Raman gain 

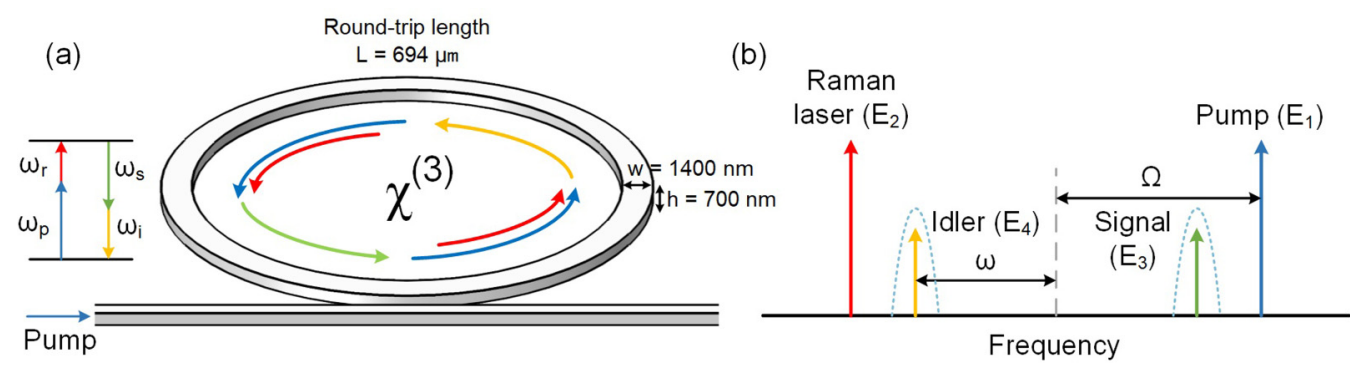

FIG. 1. (a) Schematic illustration of the AlN microresonator and the Kerr-Raman combs generation process. Signal and idler are generated by nondegenerate FWM between pump and single mode Raman laser. (b) Scheme for RFWM: the angular frequency offset between pump and central frequency is $\Omega$, and the angular frequency offset between idler and central frequency is $\omega$.

of amorphous materials on bright soliton breathers has been discussed [25], but here we investigate the breather dynamics in the presence of narrow Raman gain from crystalline materials.

In this paper, we numerically show that stable platicons can be generated in microresonators with a narrow Raman gain and normal dispersion. Since crystalline AlN has low loss at short wavelengths [33], and a narrow Raman gain spectrum with a full width at half maximum (FWHM) of $138 \mathrm{GHz}$, we design an AlN microresonator with normal dispersion near the visible band to demonstrate our RFWM based Kerr comb generation. Our numerical simulations based on the generalized Lugiato-Lefever equation (GLLE) [24,39] indicate that the AlN microresonator can support platicons and coherent visible combs using a 780-nm pump. Furthermore, our simulations show a breather platicon dynamics induced by a narrow Raman gain. The breather has a relatively low breathing frequency and exhibits out-of-phase power change between the comb lines near the pump and the lines around the Raman gain peak, which is quite different from the reported dark pulse breathers [35]. Besides the different dynamics of the Raman induced breather platicon, we find that the Raman induced breather platicons also share some universal characteristics with the Kerr effect dominated breathers. It can also transition to the chaotic breathing regime via period bifurcation when further decreasing the pump phase detuning or increasing the pump power from the stable breathing state. Our paper verifies the possibility of generating platicons in the visible band using a narrow Raman gain and adds to the study of breather dynamics.

\section{STABLE PLATICON AND VISIBLE COMB GENERATION IN AN ALUMINUM NITRIDE MICRORESONATOR}

The Kerr-Raman combs generation scheme investigated here is illustrated in Fig. 1; when Raman gain at a cavity mode exceeds loss, single mode Raman lasing is stimulated. The Kerr-Raman combs generation relies upon the RFWM process. Here, we further investigate the dynamics of coherent Kerr combs generation after Kerr-Raman combs are generated. To show the feasibility of this process in practice, we design an AlN microresonator. AlN has a narrow Raman gain spectrum, making it a suitable platform to generate broadband combs for our proposed process. The waveguide is composed of AlN-on-sapphire film, and a rectangular structure is adopted. The GVD is calculated with a finite element mode (FEM) solver; the material dispersion of bulk AlN and sapphire is obtained from Ref. [40]. We engineer the dispersion by modifying the height and width of the waveguide. Figure 2 shows the dispersion curve of the fundamental TM mode in an AlN waveguide with different geometrical parameters. The waveguide dispersion does not change significantly, even when the dimension is changed by hundreds of nanometers. The fundamental TE mode exhibits a similar dispersion curve. This suggests it is hard to engineer the waveguide geometry to achieve anomalous dispersion in the visible band.

To examine the possibility of platicon and coherent Kerr comb generation initiated via RFWM with normal dispersion, we perform simulations with parameters of an AlN microresonator the geometry of which is $1400 \times 800 \mathrm{~nm}$ [waveguide width times height, as is shown in Fig. 1(a)] using the GLLE [24,39]. Only the fundamental TM mode is propagating in the waveguide in the simulation, and there is no contribution of higher-order modes. Up to sixth order of the FEM calculated dispersion and the Raman effect are included. The GLLE can be written as

$$
\begin{aligned}
t_{R} \frac{\partial E(t, \tau)}{\partial t}= & {\left[-\alpha-i \delta_{0}+i L \sum_{n \geqslant 2} \frac{\beta_{n}}{n !}\left(i \frac{\partial}{\partial \tau}\right)^{n}\right] E+i \gamma L|E|^{2} E } \\
& +i \gamma_{R} L\left[E \int_{-\infty}^{\tau} h_{R}\left(\tau-\tau^{\prime}\right)|E|^{2} d \tau^{\prime}\right]+\sqrt{\kappa} E_{\mathrm{in}},
\end{aligned}
$$

where $t_{R}$ is round-trip time; $E(t, \tau)$ is the envelope of the intracavity field; $t$ and $\tau$ are slow and fast time, respectively; $\alpha$ is round-trip loss; $\delta_{0}$ is pump-cavity detuning; $L$ is round-trip length of the microresonator; $\beta_{n}$ is $n$ th-order dispersion at the pump frequency; $h_{R}(\tau)$ is the Raman response function, which is the inverse Fourier transform of the spectral response of Raman scattering $\tilde{H}_{R}$. The spectral response of Raman scattering has a Lorentzian shape of the form $\tilde{H}_{R}(\Omega)=\left(\Omega_{R}\right)^{2} /\left(\Omega_{R}^{2}-\right.$ $\Omega^{2}-2 i \Gamma_{R} \Omega$ ), where $\Omega$ is angular frequency, and $\Omega_{R} / 2 \pi$ and $\Gamma_{R} / \pi$ denote the central and FWHM frequency of the Ramangain spectrum [41]. $\gamma_{R}$ is the Raman nonlinear coefficient, $\gamma$ is the nonlinearity coefficient, $\kappa$ is the power coupling coefficient, and $E_{\text {in }}$ is the amplitude of the pump [39]. We consider a typical AIN microresonator where the Raman effect is calculated in the frequency domain [41], the peak of which is centered at $\Omega_{R} / 2 \pi=-18.3 \mathrm{THz}$ and the FWHM of which is $\Gamma_{R} / \pi=138 \mathrm{GHz}$ [29]. The cavity length $L=0.694 \mathrm{~mm}$ and 

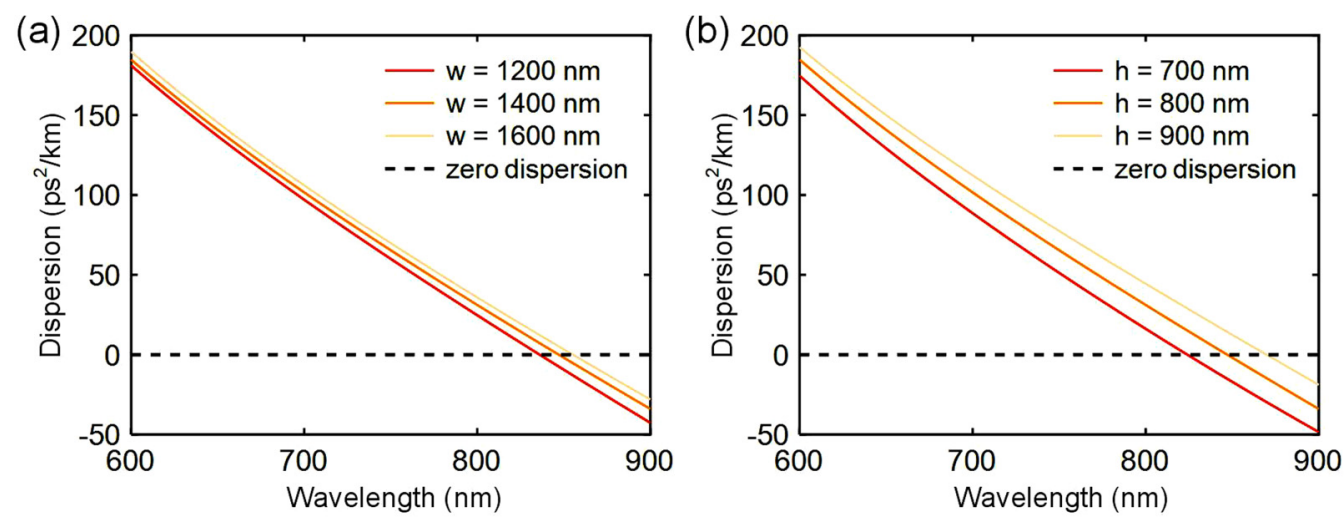

FIG. 2. Simulated group velocity dispersion (GVD) of the fundamental TM mode in the AlN waveguide with different geometrical parameters. The dispersion parameter is calculated with a finite-element mode solver. (a) Dependence of GVD on waveguide width $(w)$ when the height is $800 \mathrm{~nm}$. (b) Dependence of GVD on waveguide width $(h)$ when the width is $1400 \mathrm{~nm}$.

$t_{R}=4.93$ ps, i.e., the free spectral range (FSR) of the cavity is $203.8 \mathrm{GHz}$. The dispersion at $780 \mathrm{~nm}$ is $\beta_{2}=50 \mathrm{ps}^{2} / \mathrm{km}$, $\beta_{3}=0.22 \mathrm{ps}^{3} / \mathrm{km}$, and $\beta_{4}=-0.00012 \mathrm{ps}^{4} / \mathrm{km}$. AlN has a nonlinear refractive index $n_{2}=2.5 \times 10^{-19} \mathrm{~m}^{2} / \mathrm{W}$ [33] and the nonlinear coefficient of the waveguide $\gamma=2 \pi n_{2} /\left(\lambda A_{\text {eff }}\right)$ is calculated to be $1.438 \mathrm{~m}^{-1} \mathrm{~W}^{-1}$. The Raman nonlinear coefficient $\gamma_{R}=g_{R} \Gamma_{R} /\left(2 \Omega_{R} A_{\text {eff }}\right)=0.024 \mathrm{~m}^{-1} \mathrm{~W}^{-1}$ [41].

The pump wavelength is set to be $780 \mathrm{~nm}$, which can be obtained by frequency doubling a $1560-\mathrm{nm} \mathrm{cw}$ laser. We start by a condition where the pump power is $123.8 \mathrm{~mW}$ and detuning is -0.0072 . In this state, RFWM occurs spontaneously and the Kerr-Raman comb stably exists in the cavity; the corresponding temporal waveform and optical spectrum are shown in part I of Figs. 3(b) and 3(c). To obtain the stable platicon, we tune the pump laser in simulations with the following method: in the first $0.161 \mu$ s (corresponding to 32600 round-trips), we boost the pump to $660 \mathrm{~mW}$ with the detuning fixed to -0.0072 , as shown in the red shaded (left) region in Fig. 3(a). Then we tune the detuning linearly from -0.0072 to 0.0636 within $1.314 \mu \mathrm{s}$ (from 0.161 to $1.475 \mu \mathrm{s}$ ), which corresponds to a wavelength tuning speed of $3.54 \mathrm{pm} / \mu \mathrm{s}$, as shown in the blue shaded (middle) region in Fig. 3(a). Finally, we stop the laser tuning when the detuning reaches 0.0636 [the gray shaded (right) region in Fig. 3(a)]. The value of pump detuning is also shown with the red dashed line in Fig. 3(a).

When the pump power is boosted to $660 \mathrm{~mW}$ with a detuning of -0.0072 , the intracavity field becomes nonstationary. As the detuning increases from -0.0072 , which means the pump is rapidly tuned into the resonance, the intracavity power grows further and becomes more unstable. The waveform also becomes nonstationary and a structured single FSR comb emerges [see part II of Figs. 3(b) and 3(c)], this is similar to the spatiotemporal chaos dynamics (a)

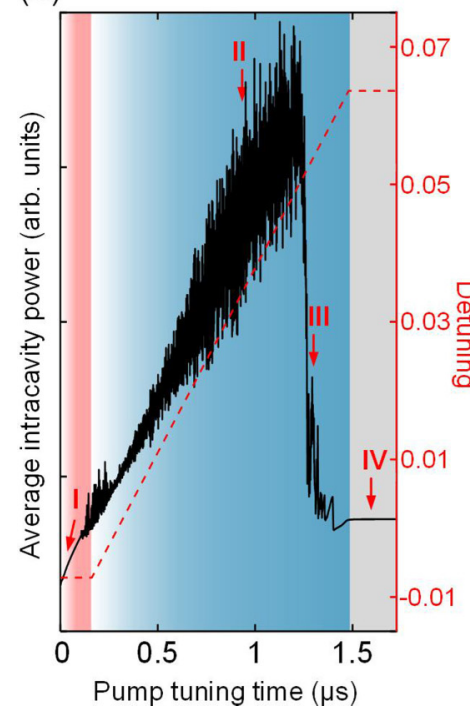

(b)

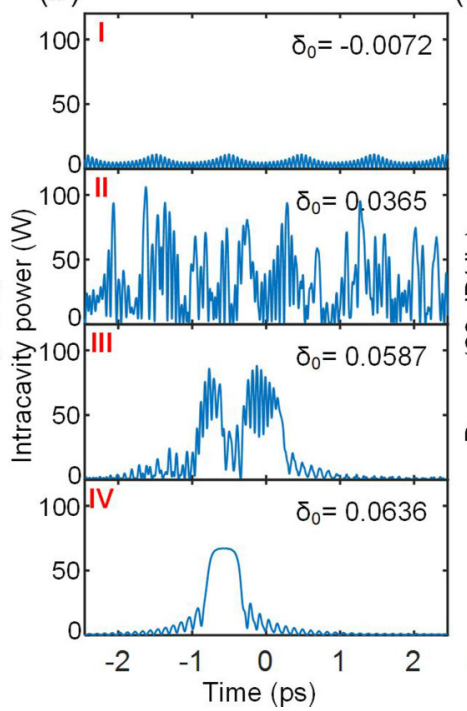

(c)

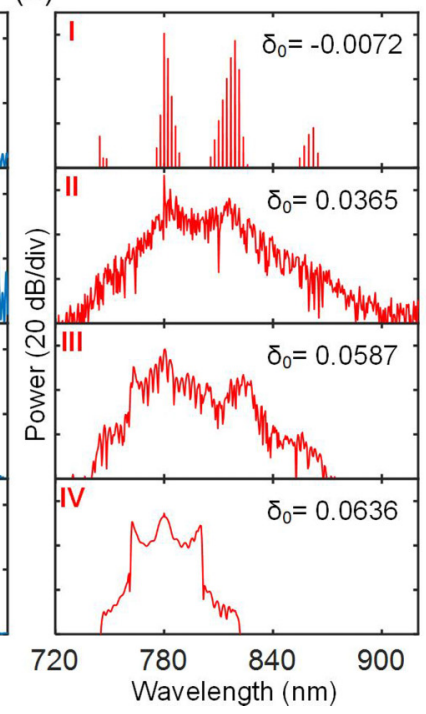

FIG. 3. Dynamics of Kerr-Raman comb generation in an AlN microresonator, the waveguide width of which is $1400 \mathrm{~nm}$ and the height of which is $800 \mathrm{~nm}$. (a) Evolution of the intracavity power when the pump is boosted to $660 \mathrm{~mW}$ and tuned into the resonance. The red dashed line shows the tuning of the detuning. In the red shaded (left) region the pump laser is boosted to $660 \mathrm{~mW}$ with a detuning of -0.0072 . In the blue shaded (middle) region the pump is linearly tuned into the resonance with detuning linearly increasing from -0.0072 to 0.0636 . In the gray shaded (right) region the pump is kept stable. (b) Temporal waveform and (c) optical spectrum for different positions I-IV in (a). 

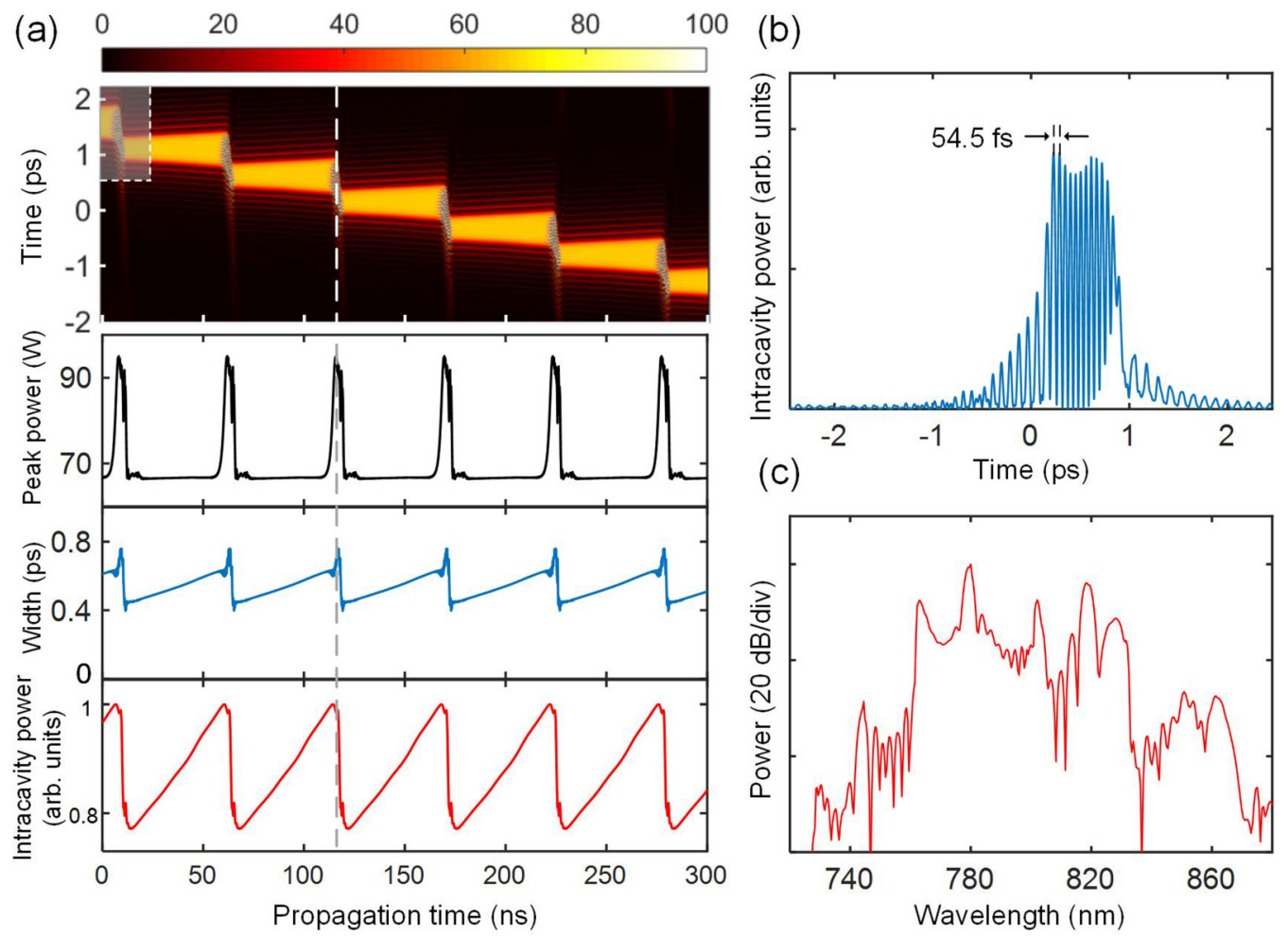

FIG. 4. Dynamics of the Raman induced breather platicon. (a) Evolution of the temporal intensity profile and the corresponding evolution of pulse peak power, pulse width, and intracavity power. (b) Temporal waveform and (c) optical spectrum of the exact moment marked by the dashed line in (a).

of the intracavity field in $\mathrm{cw}$ laser driven microresonators [34]. As the detuning is tuned to 0.057 , the intracavity power undergoes a sharp decrease, which is similar to the solitonstep formation with anomalous GVD [17]. During this power drop, part of the chaotic waveform survives and circulates in the cavity. Part III of Figs. 3(b) and 3(c) shows the waveform and optical spectrum of this state, corresponding to marker III in Fig. 3(a); a pulselike waveform starts to form but with oscillatory features.

When the detuning is tuned to 0.0636 , the Kerr-Raman comb becomes stable and the comb manifests as a stable platicon in the time domain. Part IV of Figs. 3(b) and 3(c) shows the corresponding waveform and spectrum, respectively. The comb spans more than $80 \mathrm{~nm}$ with $60-\mathrm{dB}$ power variation. A broader spectrum can be possible when using lower normal group velocity dispersion waveguide design [see the dispersion curve of $1400 \times 700 \mathrm{~nm}$ in Fig. 2(b)]. The generated platicon comb has a power of $24.4 \mathrm{~mW}$ at the output port (excluding the pump line). Although this comb power is lower than the previously reported dark soliton microcombs [23], it is higher than the power of a bright soliton microcomb, which is usually smaller than $1 \mathrm{~mW}$ [18]. Compared to the mode-interaction facilitated platicon generation [20], the formation of the platicon in our simulation is similar to the dual-pump platicon generation scheme [42]. But the primary comb lines are generated by nondegenerate FWM between the pump and the Raman laser in our scheme and stable platicon generation can be achieved with a proper detuning [42]. It is notable from part IV of Fig. 3(c) that the Raman part of the power of the stable platicon spectrum is much lower than that of other states, such as state II and state III. It can be attributed to the fact that when a stable platicon is generated the intensity of the comb lines near the pump is lower $(\approx 4 \mathrm{~dB}$ lower than state II). Thus, the Raman gain is insufficient to stimulate the modes around the Raman gain peak. This Raman-suppressing feature of the platicon spectrum also suggests that although the generated platicon is initiated by the Raman laser its characteristics are determined by the Kerr effect. Moreover, we also perform a $10^{6}$ round-trip simulation of the stable platicon evolution; the platicon shows strong stability with a constant pulse energy and pulse width during the simulation.

\section{RAMAN INDUCED BREATHER PLATICON GENERATION IN AN ALUMINUM NITRIDE MICRORESONATOR}

We also observe an intermediate state between chaotic pulse (state III in Fig. 3) and stable platicon in simulations. This state can be obtained by reducing the pump detuning while keep the pump power constant from the stable platicon state. We decrease the detuning from 0.0636 to 0.06288 , and a breathing pulse state is obtained. The evolution of the temporal intensity profile of this pulse is shown in Fig. 4(a). In this state, the width of the pulse increases while the peak power stays nearly constant during the first $5.423 \mathrm{~ns}$ of propagation in Fig. 4(a). This can be understood as the movement of two switching waves which connect the upper and lower homogenous state solutions of the bistable system [43]. Then the pulse trajectory shows a negative slope in the retarded fast time window, suggesting an increase in the group velocity. In this regime, the pulse exhibits strong oscillations in the temporal domain waveform [see Fig. 4(b)]. The 

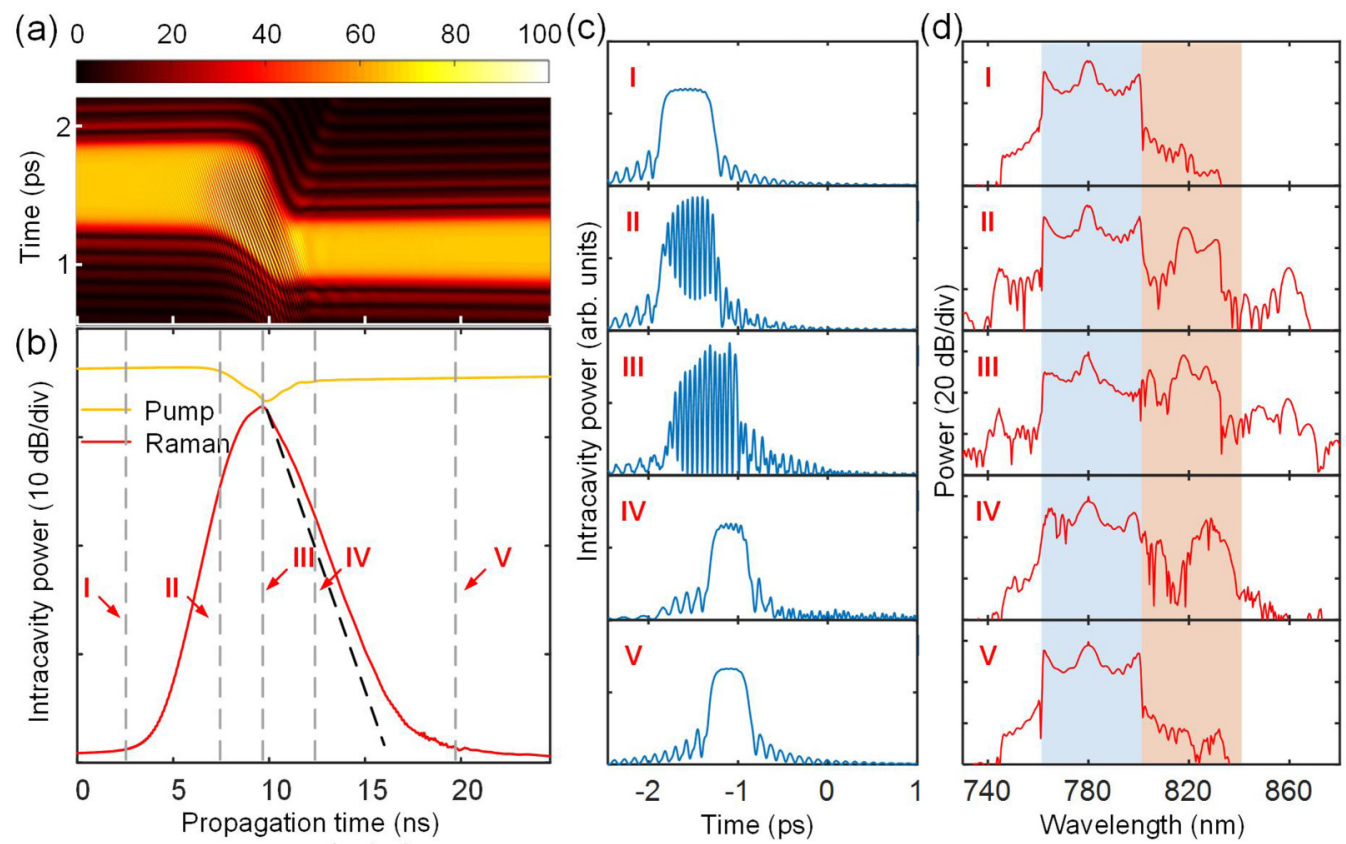

FIG. 5. Power exchange between pump area and Raman area of the platicon breather. (a) Detailed evolution of the temporal intensity and (b) the corresponding power transfer between pump area and Raman area near the negative trajectory slope regime. (c) Temporal waveform and (d) optical spectra of different times during the power transfer. The exact times of I-V are 2.465, 7.395, 9.663, 12.325, and 19.72 ns. The blue (orange) shaded regions illustrate the pump (Raman) area, corresponding to 760-800 (800-840) nm of the optical spectrum.

oscillation period is 54.5 fs, which matches the Raman gain peak frequency 18.3 THz. Associated with the oscillations on the waveform, there are strong comb lines near the Raman gain peak on the spectrum [see Fig. 4(c)]. The strong comb lines near the Raman gain peak redshift the center frequency, which induces a group velocity increase and the negative trajectory slope observed in Fig. 4(a). For the propagation time from 19.227 to $56.202 \mathrm{~ns}$, the negative slope of the pulse trajectory vanishes and the waveform becomes a flat top without oscillations again. The corresponding evolutions of the pulse peak power, pulse width, and intracavity power are shown in the lower panel of Fig. 4(a), which demonstrate a clear periodic breathing of the platicon.

To gain more insight of the dynamics in Fig. 4(a), we focus on the dynamics near the negative trajectory slope regime [the first $24.65 \mathrm{~ns}$ of propagation, marked by the gray square at top left of Fig. 4(a)]. We zoom in the temporal dynamics in Fig. 5(a). We select out the specific area in the optical spectrum and describe the power change during the evolution. We define the pump area as wavelength range 760-800 nm and the Raman area as $800-840 \mathrm{~nm}$ [see the shaded regions in Fig. 5(d)]. The corresponding power evolution of the pump area and Raman area during the transition is depicted in Fig. 5(b). In the first $5.423 \mathrm{~ns}$, the pump area power grows, thus providing more Raman gain for the Raman area. When the Raman gain exceeds the loss, the Raman area power grows rapidly at the expense of the pump area power. Meanwhile, oscillations in the time domain arise due to interference between the pump area and the Raman area. The temporal oscillation amplitude becomes larger with the increase of the Raman area power [see the representative temporal waveforms and corresponding spectra in Figs. 5(c) and 5(d)]. When the pump area power decreases further, the Raman gain decreases and the Raman area power dissipates. Oscillations in the waveform top thus become weaker and finally vanish. Meanwhile, the pump area power increases again due to the gain from input power. Note that to clearly describe the power exchange dynamics we only show the partial evolution period in Fig. 5 and the final power in Fig. 5(b) does not return to the initial condition.

In short, the whole breathing process can be described as the following steps: (1) power accumulation in the pump area, (2) power transfer from the pump area to the Raman area, and (3) power dissipation in the Raman area. There is also out-of-phase power change in the pump area and the Raman area, which appears to be similar to the FPU recurrence induced by breather solitons in microresonators with anomalous dispersion [25]. However, they are different, as in step 3 the Raman area dissipates via cavity loss instead of flowing back to the pump area. Transferring power from the Raman area to the pump area is effectively an anti-Stokes process which does not easily occur spontaneously. Indeed, the Raman area power dissipation rate essentially matches the total cavity loss rate which is indicated by the dashed black line in Fig. 5(b). Since this breather platicon dynamics is induced by the Raman effect, we classify the described phenomenon as a Raman induced breather platicon. Dark soliton breathers in the normal dispersion regime have been numerically predicted $[16,44]$ and experimentally observed [35], the dynamics of which are dominated by the Kerr effect. However, the breathing platicon observed in our simulations is induced by the narrow Raman gain spectrum and is quite different from the previously reported dark soliton breathers.

The Raman induced breather platicon predicted here also exhibits other features. First, the simulated breathing frequency (defined as the frequency of the breather 

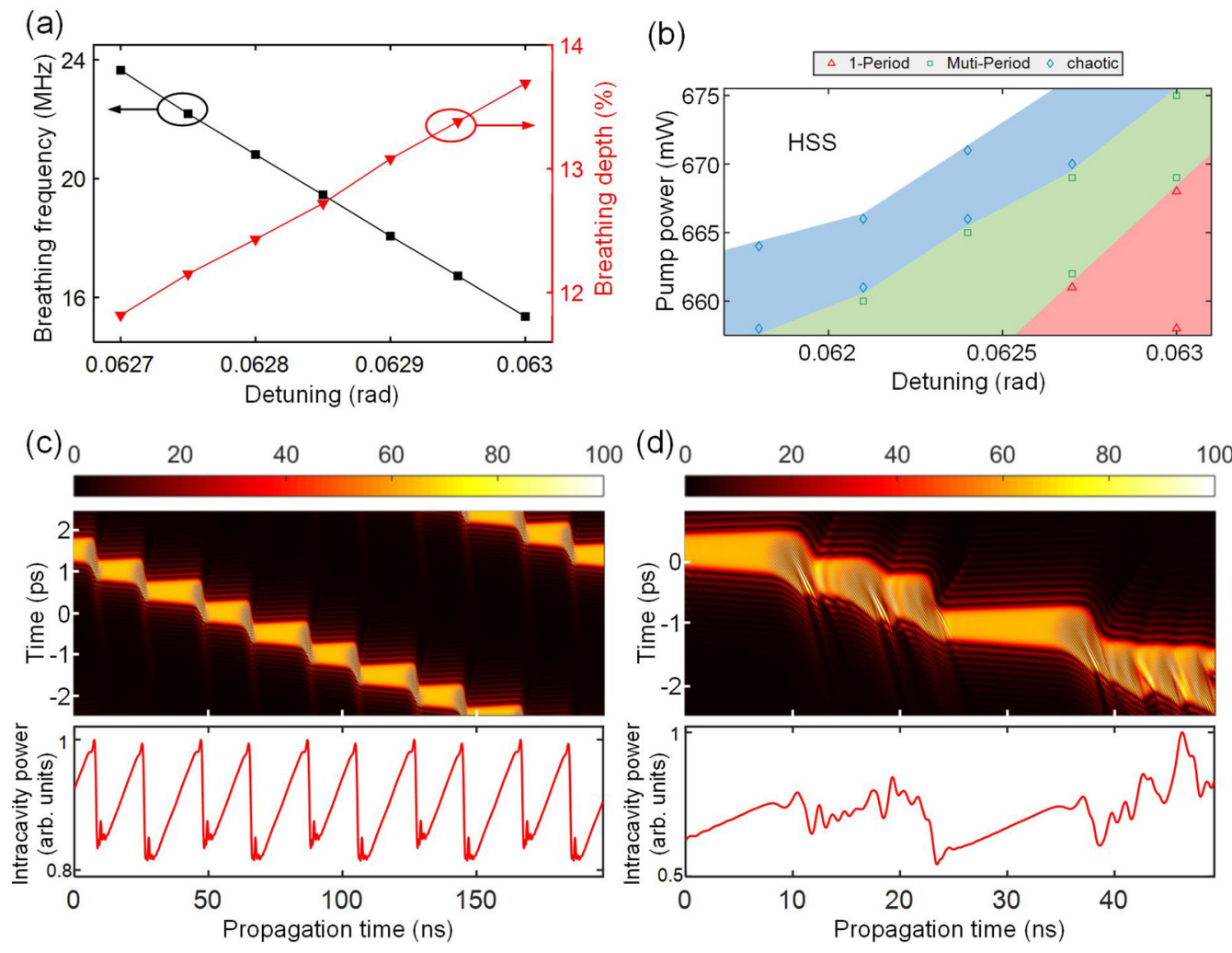

FIG. 6. Raman induced platicon breather dynamics on pump condition. (a) Dependence of breathing frequency and breathing depth on the pump detuning. (b) Phase diagram in the (pump power, detuning) plane representing different pump parameters leading to various breathing dynamics [red triangle, one-period breather; green square, multiperiod breather; blue diamond, chaotic breather; blank region, homogeneous steady state (HSS)]. (c) Evolution of the temporal intensity profile and the corresponding evolution of intracavity power of the period-doubling state when detuning is 0.0621 . (d) Evolution of the temporal intensity profile and the corresponding evolution of intracavity power of the chaotic breathing state when detuning is 0.06 .

evolving to the original waveform during propagation) is 18.7 MHz [see Fig. 4(a)], which is smaller than the half width at half magnitude (HWHM) resonance linewidth of the resonator $(192.3 \mathrm{MHz})$. However, the breathing frequencies of the Kerr effect dominated dark soliton breathers and bright soliton breathers are usually larger than the resonance HWHM [25,35]. The slower breathing is because the dissipation in step 3 occurs at the cavity loss rate and it only constitutes part of the full breathing period. Second, it shows a relatively large breathing depth [defined as $\left(P_{\max }-P_{\min }\right) /\left(P_{\max }+P_{\min }\right)$ with $P_{\max (\min )}$ being the maximum (minimum) intracavity power], which is $12.9 \%$ when the detuning is 0.06288 , more than four times that of dark soliton breathers reported in Ref. [35]. Third, this simulated breathing platicon shows a strong breathing in the waveform top (the high power part of the platicon), while dark soliton breathers show strong breathing in the waveform hole [35].

\section{TRANSITION TO CHAOTIC BREATHING}

We further investigate the influence of the pump detuning on the breathing dynamics. When we decrease the pump detuning at the breather state, the breather first shows a stable breathing period and we observe a linear increase in the breathing frequency as well as a decrease in the breathing depth, as is shown in Fig. 6(a). This is similar to the breathing dynamics of bright soliton breathers in the anomalous dispersion regime [36]. When the detuning is smaller than 0.0626 , the intracavity field begins to show a period-doubling state. Figure 6(c) shows the evolution of the temporal waveform and the intracavity power of a typical period-doubling breather platicon when the detuning is fixed at 0.0621. As the detuning is further decreased, the breather loses its periodicity and behaves as a chaotic breather. Figure 6(d) shows the evolution of the temporal waveform and the intracavity power of a chaotic breather when the detuning is fixed at 0.06 . We also investigate the effect of pump power on the breathing dynamics; we find that increasing the pump power also results in a transition to multiperiod breathing and chaotic breathing. A phase diagram describing the dependence of breathing dynamics on pump power and detuning is shown in Fig. 6(b). The blank region represents the parameter space in which only the homogeneous steady state exists in the system. The transitions from one-period breathing to multiperiod breathing and chaotic breathing via decreasing the detuning or increasing the pump power is similar to the breather dynamics in other Kerr effect dominated systems including fiber ring cavities [45] and microcavities with anomalous dispersion [36] and normal dispersion [35]. Therefore, the Raman induced breather platicons also share some universal characteristics with the Kerr effect dominated breathers. 


\section{CONCLUSION}

In conclusion, we show that RFWM is a feasible way to generate stable platicons and breather platicons in microresonators. We propose an AlN microresonator design with normal dispersion near the visible band for the generation of RFWM based Kerr combs. A 780-nm pump is used to generate stable platicons in the cavity. Breather platicon dynamics induced by Raman effect is numerically predicted. The Raman induced breather platicon shows different features from the previously reported dark soliton breathers. The influence of the pump detuning and pump power on the breathing dynamics is also investigated. Our results provide a way to achieve platicon generation using normal GVD microresonators and reveal platicon dynamics arising from the interplay with Raman gain.

\section{ACKNOWLEDGMENTS}

The authors thank Xiaosheng Xiao for insightful discussions. This research is supported by the National Key Research and Development program of China (Grant No. 2017YFF0206104) and the National Natural Science Foundation of China (Grants No. 61575106 and No. 61975090).
[1] P. Del'Haye, A. Schliesser, O. Arcizet, T. Wilken, R. Holzwarth, and T. J. Kippenberg, Nature (London) 450, 1214 (2007).

[2] A. A. Savchenkov, A. B. Matsko, V. S. Ilchenko, I. Solomatine, D. Seidel, and L. Maleki, Phys. Rev. Lett. 101, 093902 (2008).

[3] J. S. Levy, A. Gondarenko, M. A. Foster, A. C. Turner-Foster, A. L. Gaeta, and M. Lipson, Nat. Photonics 4, 37 (2010).

[4] H. Jung, C. Xiong, K. Y. Fong, X. Zhang, and H. X. Tang, Opt. Lett. 38, 2810 (2013).

[5] B. Hausmann, I. Bulu, V. Venkataraman, P. Deotare, and M. Lončar, Nat. Photonics 8, 369 (2014).

[6] X. Xue, Y. Xuan, Y. Liu, P.-H. Wang, S. Chen, J. Wang, D. E. Leaird, M. Qi, and A. M. Weiner, Nat. Photonics 9, 594 (2015).

[7] S. W. Huang, H. Zhou, J. Yang, J. F. McMillan, A. Matsko, M. Yu, D. L. Kwong, L. Maleki, and C. W. Wong, Phys. Rev. Lett. 114, 053901 (2015).

[8] M. Yu, Y. Okawachi, A. G. Griffith, M. Lipson, and A. L. Gaeta, Optica 3, 854 (2016).

[9] F. Ferdous, H. Miao, D. E. Leaird, K. Srinivasan, J. Wang, L. Chen, L. T. Varghese, and A. M. Weiner, Nat. Photonics 5, 770 (2011).

[10] S. B. Papp, K. Beha, P. Del'Haye, F. Quinlan, H. Lee, K. J. Vahala, and S. A. Diddams, Optica 1, 10 (2014).

[11] P. Marin-Palomo, J. N. Kemal, M. Karpov, A. Kordts, J. Pfeifle, M. H. P. Pfeiffer, P. Trocha, S. Wolf, V. Brasch, M. H. Anderson et al., Nature (London) 546, 274 (2017).

[12] M.-G. Suh, Q.-F. Yang, K. Y. Yang, X. Yi, and K. J. Vahala, Science 354, 600 (2016).

[13] A. Dutt, C. Joshi, X. Ji, J. Cardenas, Y. Okawachi, K. Luke, A. L. Gaeta, and M. Lipson, Sci. Adv. 4, e1701858 (2018).

[14] M.-G. Suh and K. J. Vahala, Science 359, 884 (2018).

[15] P. Trocha et al., Science 359, 887 (2018).

[16] C. Godey, I. V. Balakireva, A. Coillet, and Y. K. Chembo, Phys. Rev. A 89, 063814 (2014).

[17] T. Herr, V. Brasch, J. D. Jost, C. Y. Wang, N. M. Kondratiev, M. L. Gorodetsky, and T. J. Kippenberg, Nat. Photonics 8, 145 (2014).

[18] X. Yi, Q.-F. Yang, K. Y. Yang, M.-G. Suh, and K. Vahala, Optica 2, 1078 (2015).
[19] D. C. Cole, E. S. Lamb, P. Del'Haye, S. A. Diddams, and S. B. Papp, Nat. Photonics 11, 671 (2017).

[20] V. Lobanov, G. Lihachev, T. Kippenberg, and M. Gorodetsky, Opt. Express 23, 7713 (2015).

[21] A. F. Fercher, W. Drexler, C. K. Hitzenberger, and T. Lasser, Rep. Prog. Phys. 66, 239 (2003).

[22] A. Bartels, D. Heinecke, and S. A. Diddams, Science 326, 681 (2009).

[23] X. Xue, P.-H. Wang, Y. Xuan, M. Qi, and A. M. Weiner, Laser Photon. Rev. 11, 1600276 (2017).

[24] M. Karpov, H. Guo, A. Kordts, V. Brasch, M. H. P. Pfeiffer, M. Zervas, M. Geiselmann, and T. J. Kippenberg, Phys. Rev. Lett. 116, 103902 (2016).

[25] C. Bao, J. A. Jaramillo-Villegas, Y. Xuan, D. E. Leaird, M. Qi, and A. M. Weiner, Phys. Rev. Lett. 117, 163901 (2016).

[26] C. Milián, A. V. Gorbach, M. Taki, A. V. Yulin, and D. V. Skryabin, Phys. Rev. A 92, 033851 (2015).

[27] H. Rong, S. Xu, Y.-H. Kuo, V. Sih, O. Cohen, O. Raday, and M. Paniccia, Nat. Photonics 1, 232 (2007).

[28] P. Latawiec, V. Venkataraman, M. J. Burek, B. J. Hausmann, I. Bulu, and M. Lončar, Optica 2, 924 (2015).

[29] X. Liu, C. Sun, B. Xiong, L. Wang, J. Wang, Y. Han, Z. Hao, H. Li, Y. Luo, J. Yan et al., Optica 4, 893 (2017).

[30] Y. Okawachi, M. Yu, V. Venkataraman, P. M. Latawiec, A. G. Griffith, M. Lipson, M. Lončar, and A. L. Gaeta, Opt. Lett. 42, 2786 (2017).

[31] A. V. Cherenkov, N. M. Kondratiev, V. E. Lobanov, A. E. Shitikov, D. V. Skryabin, and M. L. Gorodetsky, Opt. Express 25, 31148 (2017).

[32] G. Lin and Y. K. Chembo, Opt. Lett. 41, 3718 (2016).

[33] X. Liu, C. Sun, B. Xiong, L. Wang, J. Wang, Y. Han, Z. Hao, H. Li, Y. Luo, J. Yan et al., ACS Photonics 5, 1943 (2018).

[34] A. Coillet, J. Dudley, G. Genty, L. Larger, and Y. K. Chembo, Phys. Rev. A 89, 013835 (2014).

[35] C. Bao, Y. Xuan, C. Wang, A. Fülöp, D. E. Leaird, V. TorresCompany, M. Qi, and A. M. Weiner, Phys. Rev. Lett. 121, 257401 (2018).

[36] M. Yu, J. K. Jang, Y. Okawachi, A. G. Griffith, K. Luke, S. A. Miller, X. Ji, M. Lipson, and A. L. Gaeta, Nat. Commun. 8, 14569 (2017).

[37] E. Lucas, M. Karpov, H. Guo, M. L. Gorodetsky, and T. J. Kippenberg, Nat. Commun. 8, 736 (2017). 
[38] E. Lucas, H. Guo, J. D. Jost, M. Karpov, and T. J. Kippenberg, Phys. Rev. A 95, 043822 (2017).

[39] F. Leo, S. Coen, P. Kockaert, S.-P. Gorza, P. Emplit, and M. Haelterman, Nat. Photonics 4, 471 (2010).

[40] M. J. Weber, Handbook of Optical Materials (CRC, Boca Raton, FL, 2002), Vol. 19.

[41] Q. Lin, O. J. Painter, and G. P. Agrawal, Opt. Express 15, 16604 (2007).
[42] M. Liu, L. Wang, Q. Sun, S. Li, Z. Ge, Z. Lu, W. Wang, G. Wang, W. Zhang, X. Hu et al., Photon. Res. 6, 238 (2018).

[43] P. Parra-Rivas, D. Gomila, E. Knobloch, S. Coen, and L. Gelens, Opt. Lett. 41, 2402 (2016).

[44] P. Parra-Rivas, E. Knobloch, D. Gomila, and L. Gelens, Phys. Rev. A 93, 063839 (2016).

[45] F. Leo, L. Gelens, P. Emplit, M. Haelterman, and S. Coen, Opt. Express 21, 9180 (2013). 